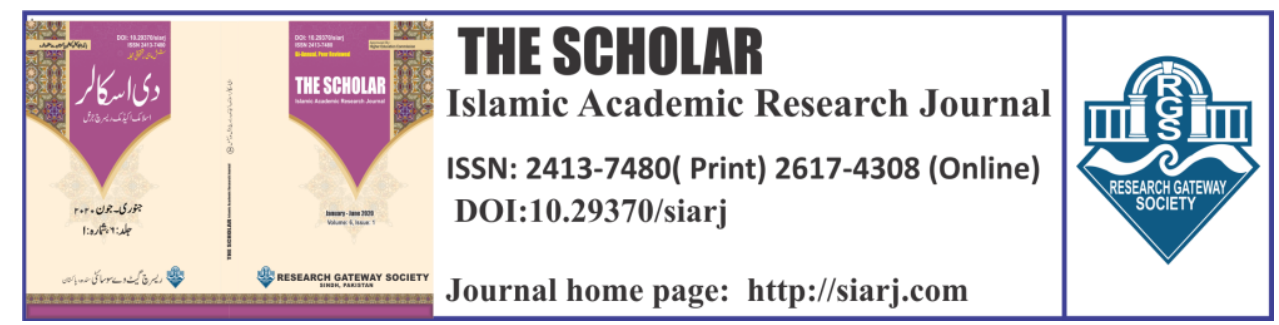

$$
\text { رؤي المفكرين المعاصرين حول إعادة صياغة القانون الإسالامي }
$$

\title{
THE VISION OF MODERN MUSLIM THINKERS ABOUT THE REFORMULATION OF ISLAMIC LAW
}

\section{1- Saifullah Bhutto}

Associate Professor, Deptt of Basic Sciences

,Quaid-e-Awam university of Eng, Science \&

Tecnology Nawabshah, Sindh, Pakistan

Email: bhuttosaifullah@quest.edu.pk

\section{ORCID ID:}

\section{https://orcid.org/0000-0001-9503-6425}

\section{To cite this article:}

Bhutto, Saifullah, and Muhammad Ajmal. "THE VISION OF MODERN MUSLIM THINKERS ABOUT THE REFORMULATION OF ISLAMIC LAW." The Scholar-Islamic Academic Research Journal 6, No. 1 (May 31, 2020): 68-86.

To link to this article: https://doi.org/10.29370/siarj/issue10ararabic4

Journal

Publisher

DOI:

URL:

License:

Journal homepage

Published online:

\section{Muhammad Ajmal}

Ph.D Scholar, Department of Islamic and

Religious Studies, Hazara University,

Mansehra

Email: ajmal13101@gmail.com

\section{ORCID ID:}

\section{https://orcid.org/0000-0002-7274-2412}

The Scholar Islamic Academic Research Journal

Vol. 6, No. 1 || Janurary -June 2020 || P. 68-86

Research Gateway Society

10.29370/siarj/issue10ararabic4

https://doi.org/10.29370/siarj/issue10ararabic4

Copyright c 2017 NC-SA 4.0

www.siari.com

2019-05-31

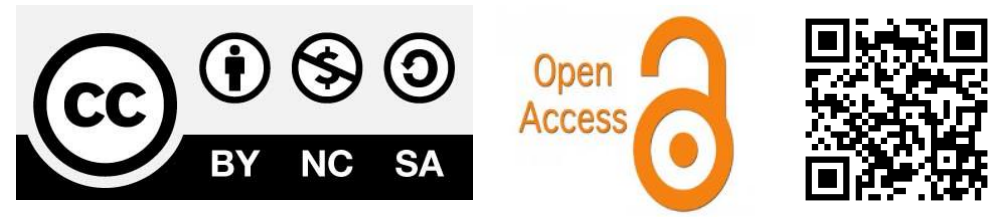




\section{رؤي المفكرين المعاصرين حول إعادة صياغة القانون الإسلامي \\ THE VISION OF MODERN MUSLIM THINKERS ABOUT THE REFORMULATION OF ISLAMIC LAW}

Saifullah Bhutto, Muhammad Ajmal

\section{ABSTRACT:}

The need to revive the Islamic viewpoint on modern issues has intensified due to major economic, social, and state structural changes. It is a big challenge for Muslim jurists \& reformists/revivalists to make important issues concordant with both the teachings of the Shariah \& the demands of the modern age. Some examples of these issues include family laws and women working, the issue of half compensation for women in case of murder, solving the issue of women travelling without blood relative (Muharram) while keeping in mind the modern means of transport. The issues related to non-Muslims like acceptance of their witness, equality of Muslim \& non-Muslim citizens in political \& social affairs. Protection of rights of Muslims living in non-Muslim countries and finding solution of problems they face. Political issues including electoral method, structures of governments. General social issues e.g. legal position and restrictions of family planning. Economic issues like interest-free banking, leasing, and devaluation of currency as a result of inflation. Medical issues like donation \& transplantation of organs, cloning etc. Beyond these, there are several judicial \& legal issues which need to be reviewed collectively through ljtihad (the Islamic mechanism of solving problems of every age with deep thought \& consideration). Some Muslim scholars have presented their opinion about these issues. We have presented in this 
paper the views of some of these modern Muslim scholars/thinkers like Sir Syed Ahmad Khan, Allamah Muhammad lqbal and Jamaluddin Afghani. These three were the progressive, moderate and reformist figures of subcontinent who played a significant role in the awakening of Muslim Ummah.

KEYWORDS: Islamic Law, Reforms, Ijtihad, Revival of Islamic Law, Allama Iqbal, Sir Syed Ahmad Khan, Jamaluddin Afghani.

$$
\begin{aligned}
& \text { الكلمات المفتاحية: الإجتهاد، الإصلاححات، القانون الإسلامي، العلامة عُمَّمَ إقبال، سر } \\
& \text { سيد أحمد خان، جمال الدين الأفغاني، صياغة. }
\end{aligned}
$$

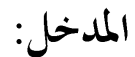

$$
\begin{aligned}
& \text { الحمد لله الذى جعل في كل زمان فترة من الرسل، بقايا من أهل العلم، يدعون من ضل } \\
& \text { إلى الهدى'، و يصبرون منهم على الأذى'. يجيون بكتاب الله الموتى'، ويبصرون بنور الله أهل } \\
& 1 \\
& \text { العمى. } \\
& \text { والصلوة و السلام على رسوله الكريم الذي قال: "يحمل هذا العلم من كل خلف عدوله، } \\
& \text { ينفون عنه تحريف الغالين و تأويل الجاهلين و انتحال المبطلين". } 2 \\
& \text { وقد هيأ الله تعالى لهذه الأمة علمائها لقيادة الأمة ،خاصة عند الملمات، فاختار لها من } \\
& \text { العلماء العاملين من يقودهم و يرشدهم إلى الحق، لتكون حجة الله قائمة بهم إلى قيام }
\end{aligned}
$$

${ }^{1}$ Al-Imam Ahmed bin Hanbal, Muqaddamah Al-radd Alal Jahmiyah -wal Zinadiqah, Tehqeeq, Abdul Rehman, Darul liwaa, Riyaz, 1397. AH. p. 58.

2 Al-Aajir, Al-Imam Abu Bakr Muhammad bin Al-Hussain, Al-Sharia, Ansar ul Sunnah, Lahore, P. 20. 
الساعة، فهم وراثو النبوة، وهم النجوم يهتدى بها في الظلمات، فقيض الله لدينه الخاتم عند رأس كل مئة عام من يقوم بتجديد معالمه، وإحياء ما اندرس منه. وقد قام العلماء العاملون بهذه المهمة (بحديد الدين) حق القيام منذ فجر الإسلام، و إلى اليوم، مدافعين عن الحق، ينفون عنه تحريف الغا لين، وتأويل الجاهلين، و إنتحال المبطلين. و الأمة الإسلامية اليوم تعيش على مفترق الطرق، حيث تكالبت عليها أمم الكفر و الشرك. ناهيك عما اصاب الأمة من داخلها -اذ تفرقت إلى شيع و أحزاب، و مذاهب، و جماعات مفترقة، وإفتراق و إختلاف. كما ظهرت بعض الإبحاهات لتجديد الدين من بعض العقلانيين و العصرانيين نادوا به على ضوء أصول و قواعد ارتضوها و وضعوها لأنفسهم فنحوا بالتحديد منحى آخر لم

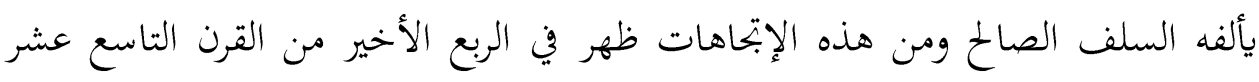
الميادي، الإتحاهات العقلانية والعصرانية والحداثية وغيرها، توجد هذه الإتحاهات لدى كثير من المفكرين و الأدباء والمثقفين والدعاة الحزبيين و غير الحزبيين. 1. ظهورالعصرا نية* في الفكر الإسلامي الحديث:

عاشت أوربا عصر النهضة الحديثة في مطلع القرن التاسع عشر الميلادي في كل مناحي الحياة السياسية والإجتماعية والإقتصادية، مقابل ما منت به الدول العربية والإسلامية بكثير من التدهور والإنحلال والتفكك واعقب ذلك إرسال البعثات العلمية خاصة إلى البلاد الغربية، وإرسال خبراء و أساتذة للتدريس في الجامعات العربية، حيث تأثر كثير من

"العصرانية مصطلح حادث و هي حكة تجديدية ظهرت في العصر الحديث في الغرب في الديانة اليهودية النصرانية،

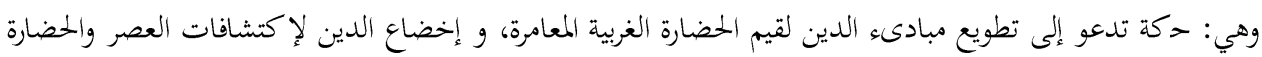

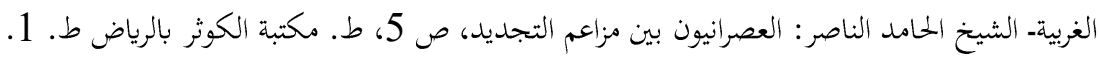


المثقفين والمفكرين من أبناء المسلمين بما عند هؤلاء الأوربيين، وكان من أشهر أولئك

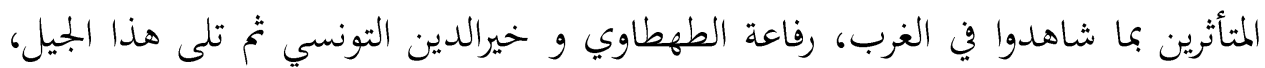

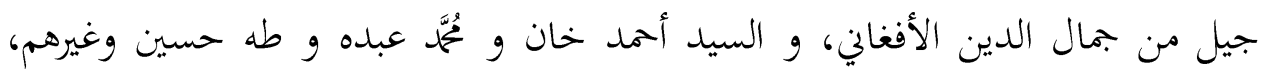

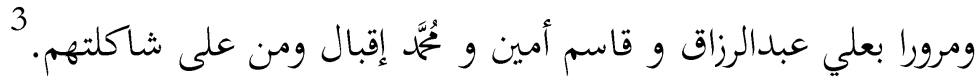

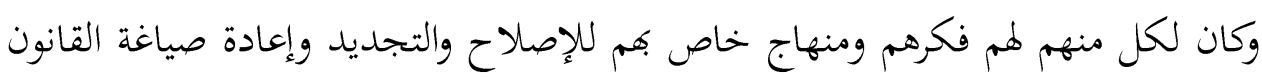

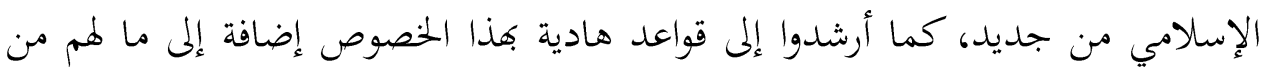

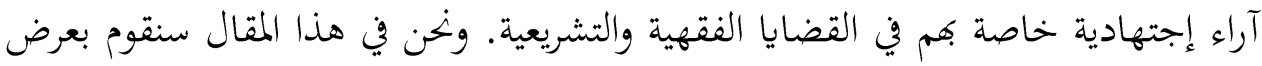

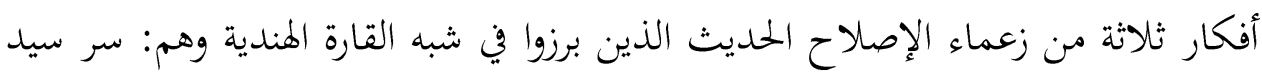
أحمد خان و جمال الدين الأفغاني و ومُجمَّم إقبال. 2. سر سيد احمد خان: ولد سر سيد أحمد في دهلي في أسرة من علية القوم عريقة وذات التال

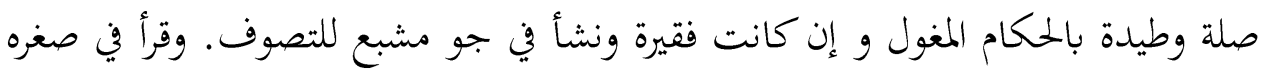

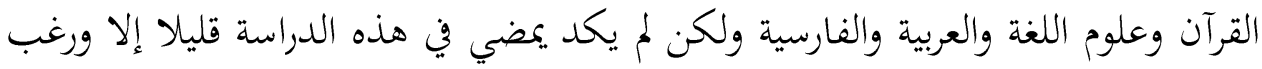

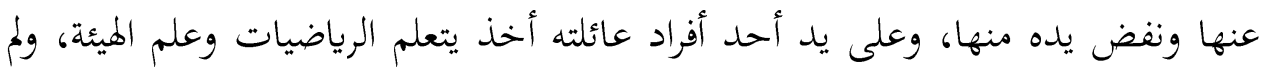

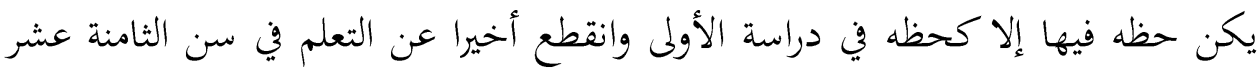
وعاش في شبابه حياة مرح يحضر حفلات الرقص والغناء الشائعة في طبقته. 4 وفي سن الثانية والعشرين اضطر سبب وفاة والده للإلتحاق بخدمة الحكومة الإنجليزية في

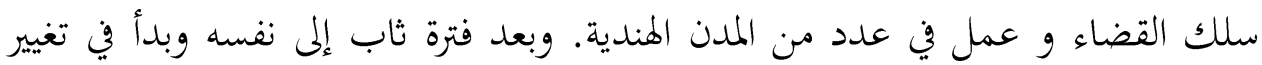
حياته وإصلاحها و أقبل على التعلم من جديد، ثم ألف عددا من الكتب في السيرة النبوية

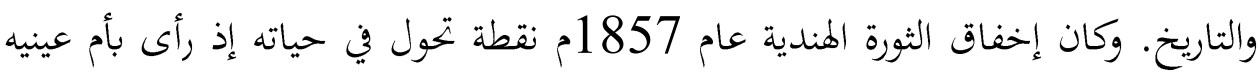

3 Abdul Aziz Mukhtar Ibrahim, Al-Asranyoon wa al-Mafhoom li Tajdid din, Maktabah Rushd, Riyaz. vol. 1, P. 26-27,

${ }^{4}$ Danish Gah Punjab, Urdu, Dairatul Maarif Islamiah, Department of Urdu, University of Punjab, Lahore, 2006, P. 4, 114-115. 
المأساة التي عاشها المسلمون وسقوط دولتهم الخراب والدمار الذي لحقهم. ومنذ أول وهله كان يدرك ان تلك الثورة نتيجتها الفشل فلهذا وقف في أثناءها يناصر الإنجليز ويساعد في حمايتهم. لقد أيقن أن ولاء المسلين للحكم الإنجليزي هو السبيل الوحيد لإنقاذهم، ولم يكن ذلك موفقا سياسيا فحسب بل كان نابعا من إعجابه المفرد بالإنجليز وبحضارتم وعلومهم. وهذا جعل نصب عينيه منذ تلك اللحظة هدفا سعي لتحقيقه طول حياته، وهو أن يقلد المسلمون الإنجليز والحضارة الغربية في كل شيء مما جعل "جون ميكدونالد" الذي كان محررا آنذاك في إحدى صحف إله آباد يصفه وزمره التي بدأت تلتف حوله من المسلين بأفم "إنجليز في كل شيء بإستثناء العناصر الأساسية لعقيدتم الدينية". 5

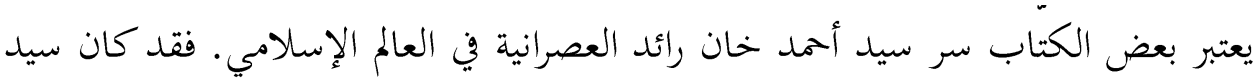
أحمد أول رجل في الهند الحديثة يتحقق من ضرورة وجود تفسير جديد للإسلام تفسير تحرري وحديث وتقدمي ولم يكن سيد أحمد خان أول ممثل للنزعة العصرانية فحسب بل كابل كان نموذجا كاملا بها و كل الذين جاؤا من بعده لم يضيفوا شيئا جديدا بل كانوا يريدون صياغة أفكاره بصورة أو أخرى. و إذا أمعنا النظر في تعريف العصرانية التي هي في جملتها محاولة للتوفيق بين الدين والعصر الحديث بإعادة تأويل الدين و تفسير تعاليمه في ضوء المعارف العصرية الساعدة، فإن هذا التفريق برمته ينطبق على المدرسة الفكرية التي أنشأها سيد أحمد خان، تلك المدرسة التي قامت على أساس تقليد الحضارة الغربية وأسسها المادية وإقتباس العلوم العصرية بحذافيرها وعلى علاتما، وتفسير الإسلام والقرآن تفسيرا يطابقان ما وصلت إليه المدنية والمعلومات الحديثة في آخر القرن التاسع عشر المسيحي، ويطابقان

${ }^{5}$ Bistami, Muhammad Saeed, Mafhoom Tajdid din, Darul Dawat, Kuwait, 1984, P. 120. 
رؤي المفكرين المعاصرين حول إعادة صياغة القانون الإسلامي

هوى الغربين وآرائهم وأذواقهم والإستهانية بما لا يثبته الحس والتجربة ولا تقرره علوم الطبيعة في باديء النظر من الحقائق الغيبية. 2.1

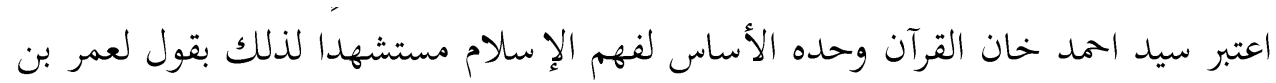
الخطاب "حسبنا كتاب الله" وفي ضوء الظروف الجديدة وتوسع المعروف الإنسانية لا يمكن الإعتماد في فهم القرآن على التفاسير القديمة وحدها التي اشتملت على كثير من الخرافات، ولكن ينبغي الإعتماد على نص القرآن وحده الذي هو بحق كلمة الله، ومن خلال معرفتنا

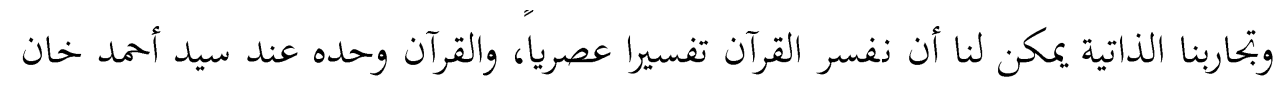

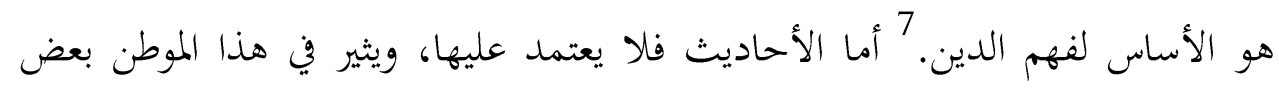

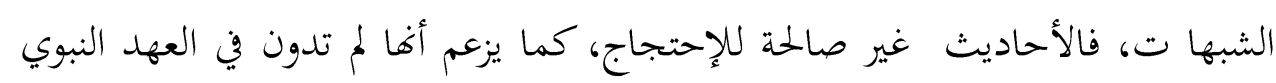
بل دونت في القرن الثاني من الهجرة في عصر مضطرب بالصراعات السياسية والإختلافات

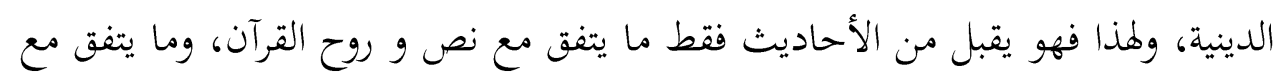

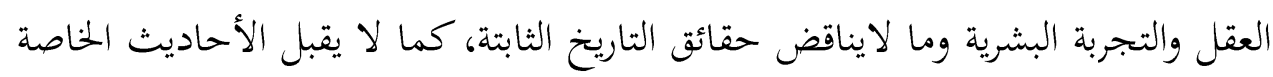
بالأمور الدنيوية التي تشمل المسائل السياسية والإدارية والإجتماعية والإقتصادية. ويظهر بأن الشبهات التي أثارها سيد أحمد خان حول الحديث ومؤلفاته لا تختلف كثيرا في النهاية ما وصل إله المستشرقون أمثال جولد تسهير وجوزف شاخت آنست. 8 ويسخر سيد خان من أولئك الذين يظنون أن كل شيء قد اكتمل على أيدي الفقاء

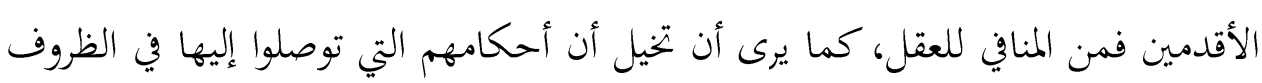

${ }^{6}$ Ibid. p. 121-122.

7 Islahi, Zafarul Islam, "Sir Sayed Aor Ijtihad", Monthly Urdu Dunya, Delhi, Nov. 2002, P. 17; Pani Patti, Shaikh Muhammad Ismail, Maqalat Sir Sayed, Majlis Taraqqi Adab, Lahore, 1962, vol. 13, P. 39.

${ }^{8}$ Muhammad Akram Shaikh, Moj Kosar, Idarah Thaqafat Islamiah, Lahore, 1997, P. 156-168. 
رؤي المفكرين المعاصرين حول إعادة صياغة القانون الإسلامي

الخاصة بأيامهم صالحة أيضاً لعصرنا الحديث، وذالك لأن حاجات عصرنا في رأيه تختلف

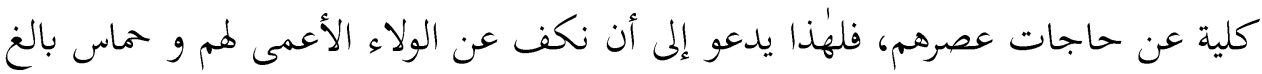

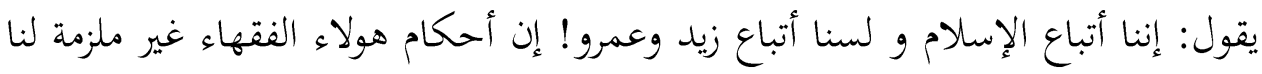

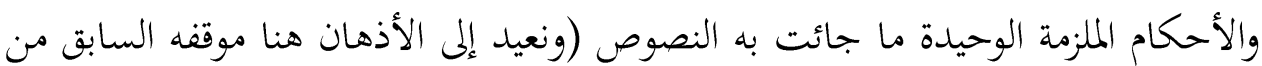
ولا يرفض سيد أحمد خان قبول آراء الفقهاء السابقين في صورتحا الفردية فقط بل حتى ولو

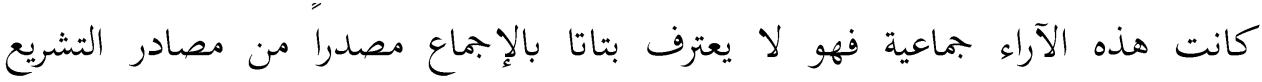
الإسلامي. إن باب الإجتهاد في نظره مفتوح في كل المسائل ولا يجب تقييده بآراء بجموعة من الفقهاء

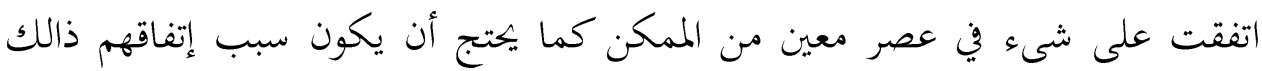

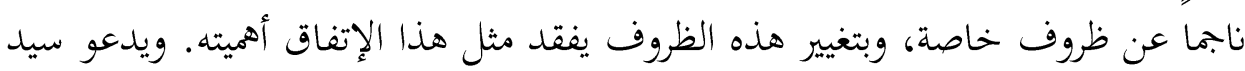

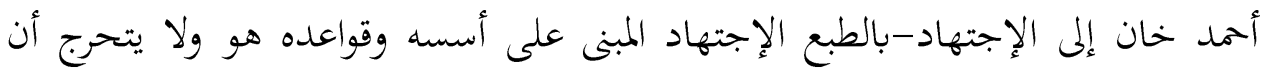

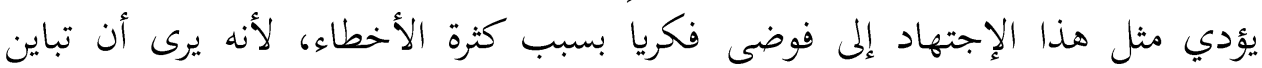
وجهات النظر والحرية الواسعة هي الوسيلة الوحيدة لتقدم الأمة. 9

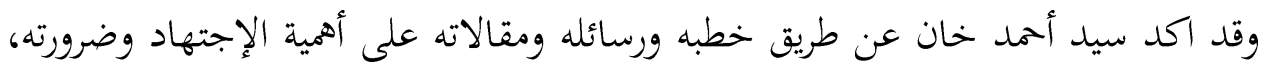
فيقول في إحدى مقالاته عنواها:"حاجة أهل السنة والجماعة إلى مجتهد" يقول ما معان معناه:

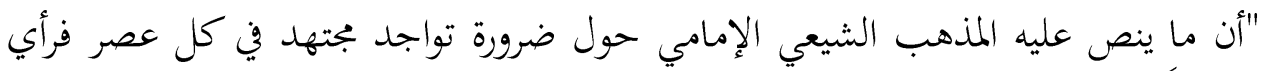

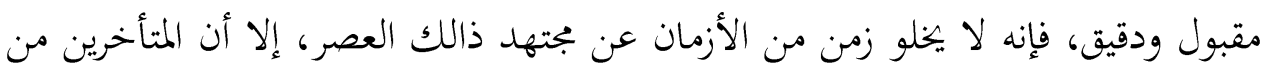

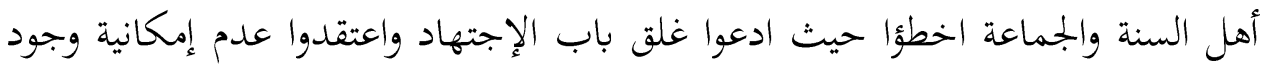

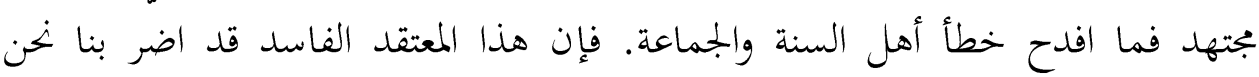

\footnotetext{
${ }^{9}$ Bistami, Muhammad Saeed, Mafhhom Tajdid Din, P. 121-122.
} 
رؤي المفكرين المعاصرين حول إعادة صياغة القانون الإسلامي

المسلمين أيما ضرر سواء في الدين أو في الدنيا، فالواجب علينا اذا: أن نقوم بالبحث والتدقيق في جميع القضايا الدينية منها والدنيوية، فالحياة الإنسانية دائما عرضة للمستجدات والنوازل، واذا افترضنا عدم وجود علماء بجتهلين من الأحياء فهل ترجع الأمة عند حدوث قضايا جديدة إلى من انتقلوا إلى رحمة الله! ولذا وجود بجتهدين في كل عصر وزمان أمر لازم". 10 ونتخلص إلى أن سيد أحمد خان يبالغ في التأكيد على ضرورة الإجتهاد بالرأي المُض ويعتبره من الحقوق الأساسية لكل مسلم يجب عليه ممارسته، أما بالنسبة للتراث الحديثي فينظر إليها نظرة ارتياب وتشكيك ويقرر كما اسلفنا بأن الأحاديث ليست موضع ثقة

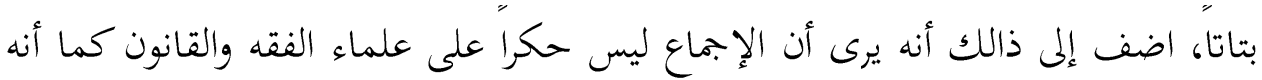
أراد من وراء كتاباته أن يثبت أن حقائق الإسلام وتعاليمه لاتتعارض مطلقا مع قوانين الطبيعة وما وصلت إليه المدنية والحضارة الغربية لأن القرآن هو كلمة الله وقوانين الطبيعة هي فعل الله ولايتعارض كلامه مع فعله، ومن أجل ذلك الهدف وضع تفسيرا خالف فيه كالام العرب وآراء السلف وإجماعهم لمحاولته تأويل ما ظنه تعارضا بين كالام الله وبين قوانين الطبيعة 11، ويتضح لنا من فكر سيد أحمد خان وآرائه صورة فجه وطريقته للمالائمة بين الإسلام والعصر الحديث. وقد أصبح منهجه ذاك مدرسة فكرية تأثر بها تلاميذه و خلفائه إلى اليوم، ومن أشهر هؤلاء التلاميذ، شراغ علي وأمير علي، وخدا بخش الشاعر، غلام أحمد برويز، وخليفته عبدالحكيم، ومولانا مُحُّجّ علي، أحد قادة حركة الأحمدية. وهؤلاء الخلفاء حملوا فكره واتبعوا طريقته، وهم نماذج لمدرسة العصرانية ودعاة التحديث في الهند.

${ }^{10}$ Aziz Ahmed, Barr-e-Saghir Men Islami Jadidiat, P. 85.

${ }^{11}$ Ibid. p. 85.

${ }^{12}$ Pani Patti, Shaikh Muhammad Ismail, Maqalat Sir Sayed, P. 290-291. 
رؤي المفكرين المعاصرين حول إعادة صياغة القانون الإسلامي

3. جمال الدين الأفغاني: مُحَّل بن صفدر الحسيني جمال الدين، فيلسوف الإسلام في عصره وأحد الرجال الأفذاذ الذين قامت على سواعدهم كضة الشرق الحاضرة. ولد في أسعد آباد (أفغانستان) ونشأ بكابل. وتلقي العلوم العقلية والنقلية وبرع في الرياضيات وسافر إلى الهند وعاد إلى وطنه فأقام بكابل وانتظم في سلك رجال الحكومة في عهد دوست تُمَّم خان تم رحل مارا بالهند ومصر وأقام في مصر ونفخ فيها روح النهضة الإصلاحية في الدين والسياسة وتتلمذ له نابغة مصر الشيخ تُمَّل عبده وكثيرون. وتوفي سنة 1363هـ وكان عارفا باللغات العربية والأفغانية والفارسية والسنسكرية والتركية، وتعلم الفرنسية والإنجليزية والروسية، وإذا بالعربية فلغته الفصحى. واسع الإطلاع على العلوم

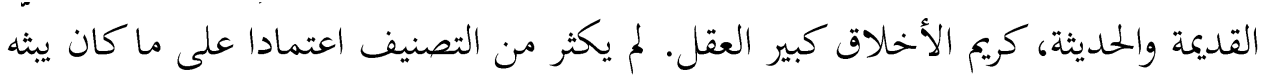
في نفوس العاملين وانصرافا إلى الدعوة بالسر والعلن. 13 إن حركة جمال الدين الأفغاني هي في واقع الأمر امتداد للكفاح الإسلامي في تاريخ الجماعة الإسلامية وليست حركة منعزلة عن غيرها، أنّ أساسها كأساس آية حركة كفاحية إسلامية ماضية، وغايتها ذات الغاية بهذه الحركات جميعها. يدعو الأفغاني إلى الترابط الوثيق بين المسلمين وغير المسلمين في الأوطان الإسلامية وإلى عدم التميز بين مسلم وغير مسلم -فتبدو دعوته هذه في مظهر الشرقية أو الوطنية- تبدو سياسية، ولذلك يميل بعض المؤرخون لجمال الدين إلى أن يسمى حركته هذه حركة الشرقية. ولكن في دعوته هذه هو مسلم، وعمله عمل إسلامي لأنه يستند إلى الإسلام في تاريخ الفتوح، وفي تعاليمه في الصلة بين المسلم وغيره، سواء في مكان واحد أو مكانين مختلفين يدعو إلى نبذ الخصومة بين الشيعة والسنية، يؤلف بين سلطنين قويتين في رقعة العالم الإسلامي إذ ذاك! بين سلطة إيران وسلطة القسطنطينية بعد ذهاب دولة الهند

${ }^{13}$ Al-Zirkili, Khairuddin, Al-A 'alam, Darul Ilm Lilmalayeen, Beirut, 1984, vol. 6. P. 126. 
رؤي المفكرين المعاصرين حول إعادة صياغة القانون الإسلامي

الإسلامية، فيبدو لذالك سياسيا أو وسيطا في بجال السياسة، ولكنه يدعو بدعوة السلام

في ذالك.

أما عن المصادر الشريعة فيقول: القرآن وحده هو سبب الهداية، والعمدة في الدعاية وما تراكم عليه وبتمع حوله من آراء الرجال واستنباطاتم ونظرياتم فينغي أن لا نعول عليه كوحي، وإنما نستأنس به كرأي .....ولا نحمله على أكتافنا مع القرآن في الدعوة إليه، وإرشاد الأمم إلى تعاليمه ....وتفسيره واضاعة الوقت في عرضه. 15 وبهذا يكون هناك عنده مصدر مزكد: هو القرآن، و ما في منزلته من السنة المتواترة، والإجماع وأعمال النبي المتواترة إلى اليوم هي السنة الصحيحة التي تدخل من مفهوم القرآن، والدعوة إلى القرآن وحده. و مصدر غير مزٔكد: يصح أن يستأنس به، ولكن يجب أن لايؤخذ به، وهو ما بحمع حول القرآن من آراء المسلمين و شروحه للإسلام. 16 و يقول: إن الإجماع هو إجماع الصحابة رضي الله تعالى عنهم على عمل أو أمر من أمور

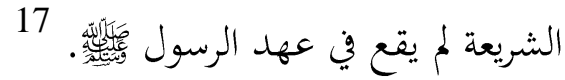
أما بالنسبة للإجتهاد: فيذكر البرت هورانى عن فكرته حول استمرارية الإجتهاد، حيث يقول: أن باب الإجتهاد لم يغلق قط وأنه يجق لأهل العلم بل يجب عليهم أن يمارسوا تطبيق تعاليم القرآن بخصوص القضايا المعاصرة التي تعرض لمجتمعهم، وإهمال ذلك الواجب يؤدي إلى ماساة التقليد والجمود الفكري، بل قد يعتبر ذلك المعاداة للدين الإسلامي الحقى، تماما 18

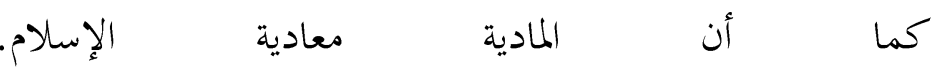

14 Al-Bahi, Muhammad, Al-fikr Islami Al-hadith wa silatuhu Bil-Ist'imar al-Gharbi, Maktabah wahbatul A'bidin, 1964, P. 84.

${ }^{15}$ Ibid. p. 84

${ }^{16}$ Ibid. p. 84

${ }^{17}$ Ibid. p. $84-85$

18 Albert Hourani, Arabic Thought in the liberal Age 1798-1939, Oxford University Press, London, 1967, P.127. 
نرى جمالدين الأفغاني: يحمل على السيد أممد خان و ينقد ابتاهه الطبيعي نقدا مراً في

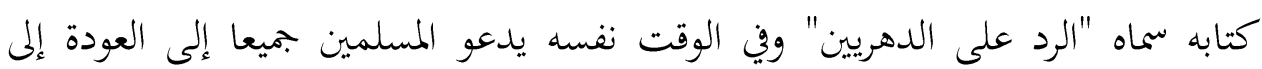

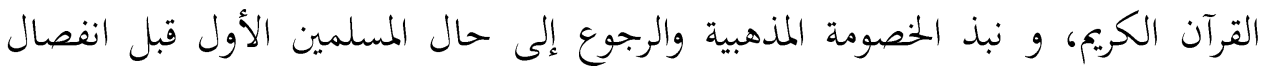

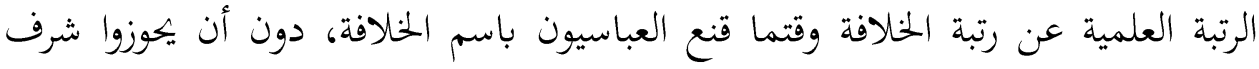
العلم و التفقه في الدين والإجتهاد في أصوله و فروعه، كما كان الراشدون رضى العيدة الله عنهم.

مات جمال الدين في سنة 1897م- بعد صراع عنيف مع الإستعمار الغربى استمر قرابة

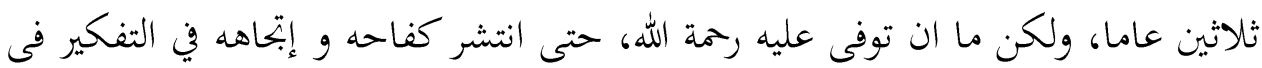

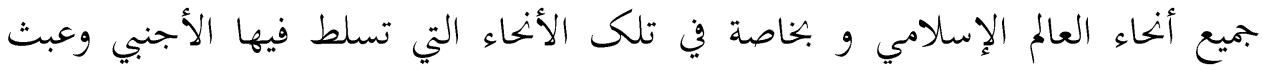
بمقدسات المسلمين و بكراماقم واقتصادياقم ومواردهم في الثروة الطبيعية.

$$
\text { - مات جمال الدين في إستامبول و ظهر أثره: }
$$

- و في الجزائر: في جميعة علماء الجزائر المؤسسها المرحوم عبدالحميد بن باديس المتوفى

${ }^{19}$ Al-Bahi, Muhammad, Al-fikr Islami Al-hadith, P. 85-86

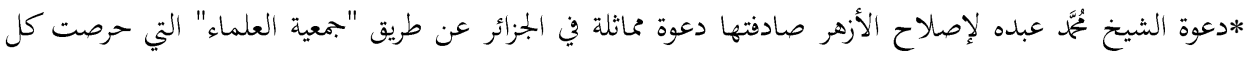

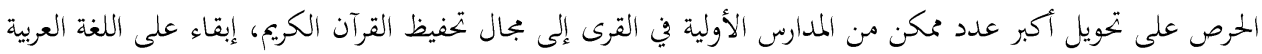

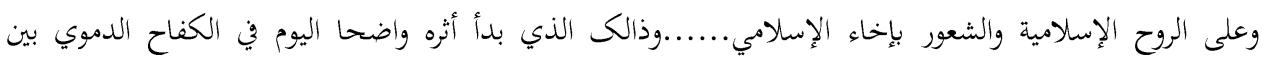

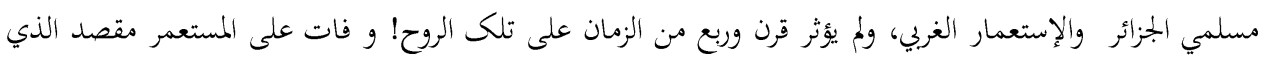

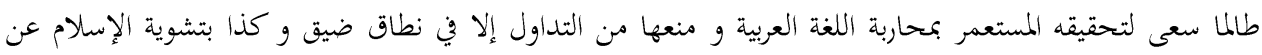

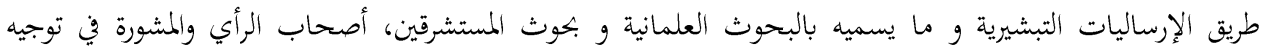

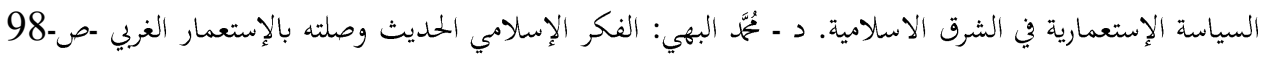

.99. 
- و في إندونيشيا: في حركة تجديد المنار.

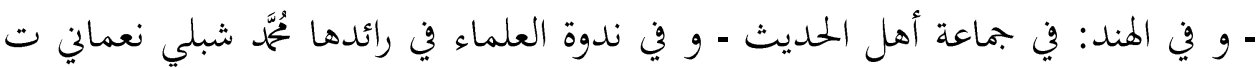

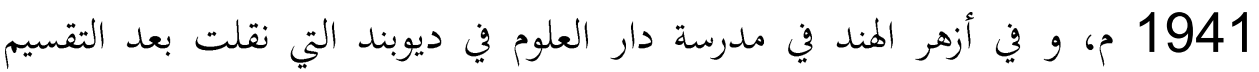
1947م إلى باكستان.

و كل هذه الحركات نجد لديها هدفا واحدا: هو تحرير الوطن الإسلامي من الإستعمار

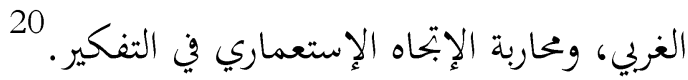

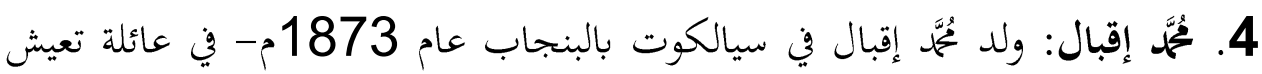
على الزراعة- نزح جدها الأكبر عن كشمير.

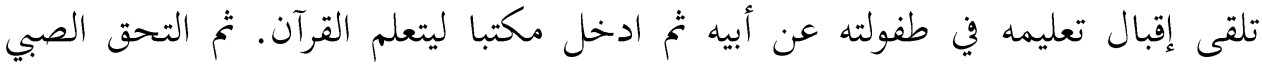

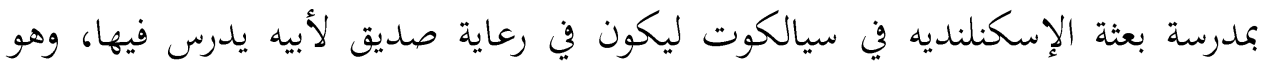

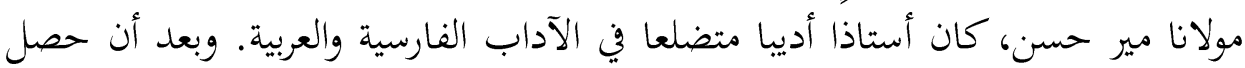

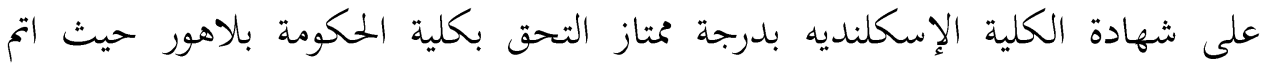
دراسته. وهناك تتلمذ على المستشرق الكبير سير توماس رأنولد. ومن هذه الكلية حصل

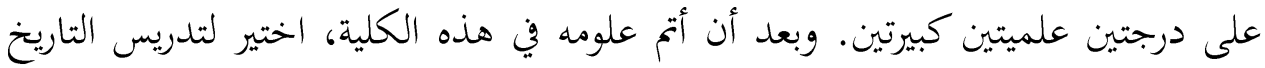
والفلسفة في الكلية الشرقية بلاهور، ثم نصب لتدريس الفلسفة واللغة الإنجليزية بكلية الحكومة التي تخرج فيها.

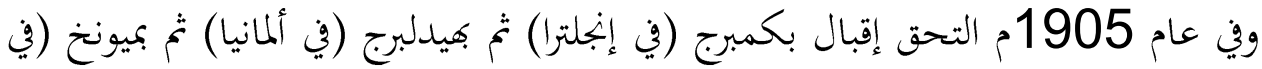

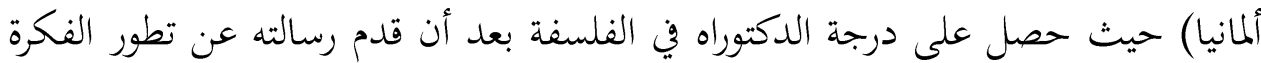

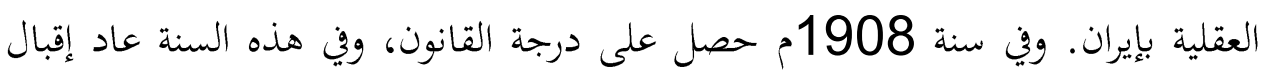
إلى وطنه.

\footnotetext{
${ }^{20}$ Ibid. p. 94
} 
ومع أن إقبال كان شاعرا وفلسوفا، إلا أنه لم يقطع صلته بالسياسة، فكان عضوا بالملس

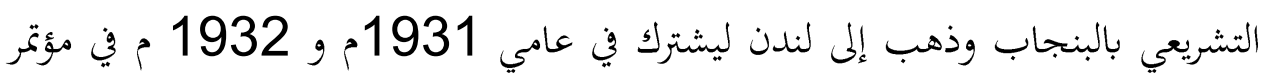

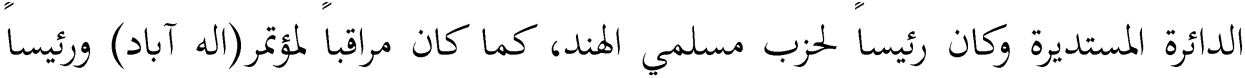

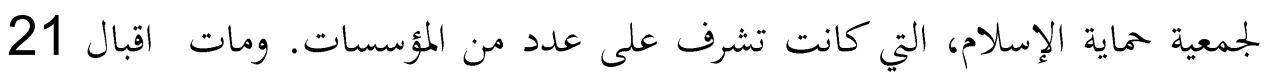
ابريل سنة 1938م ودفن بلاهور. 21 و إقبال هو أول من نادى بضرورة انفصال المسلمين عن الهندوس، ووجوب تكوين دولة

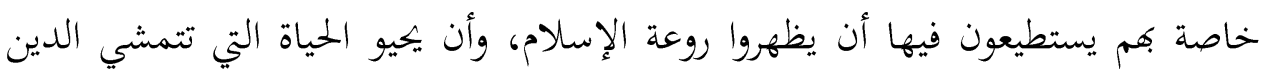

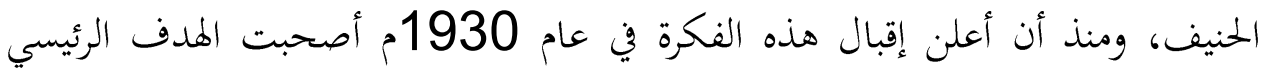

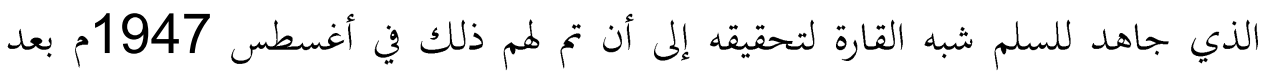
22

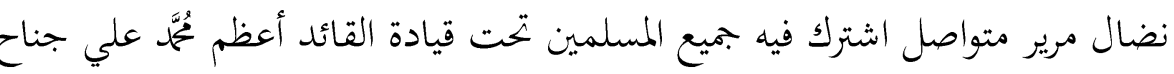

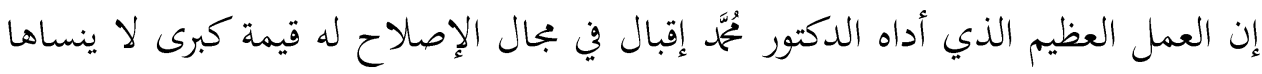

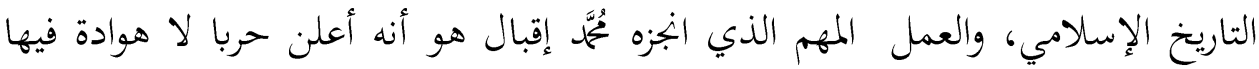

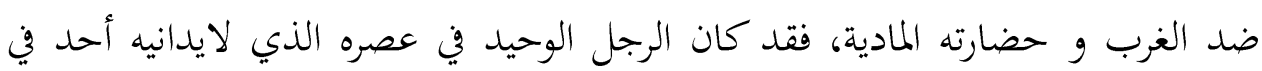
تعمقه في فلسفة الغرب ومعرفته بحضارته وحياته، فلما غضض يفند فلسفته و أفكاره المادية 23 بدأ يذوب سحر الحضارة الغربية الذي كان يبهر القلوب ويستولى على النفوس.

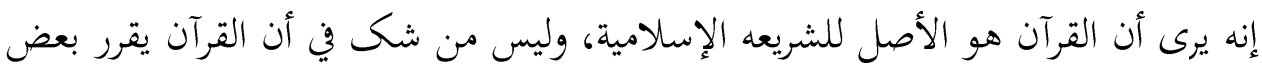

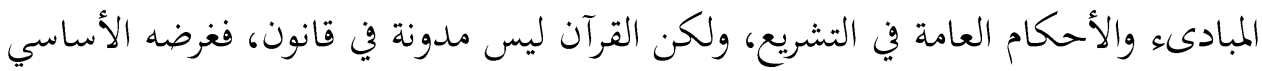

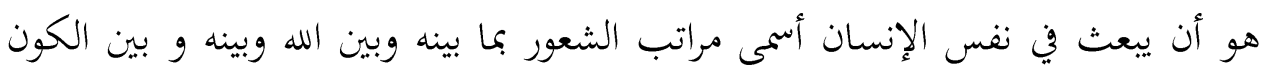

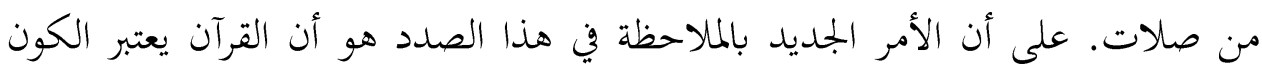

${ }^{21}$ Danish Gah Punjab, Urdu, Dairatul Maarif Islamiah vol. 16, p. 73.

${ }^{22}$ Sifaratul Bakistaniah, Al-Qahirah, Nashria Iqbal, Cairo, 1956.

${ }^{23}$ Muhammad Iqbal, Dr. Tashkeel Tajdeed. Al-Ilahyat al- Islamiyah, p. 26; Muhammad Khalid Masood, Dr. Iqbal ka Tasawwur Ijtehad, P. 146-147. 
متغيرا، ومن الواضح الجلي أن القرآن بما له من هذه النظرة لا يمكن أن يكون خصما

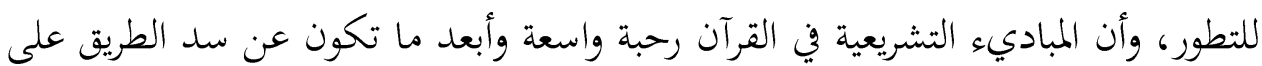

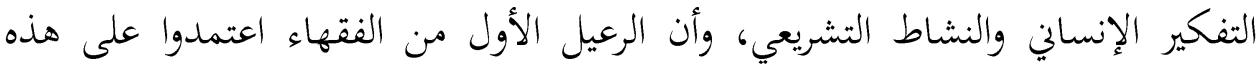

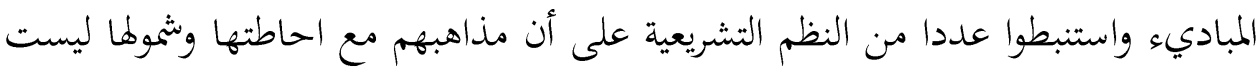

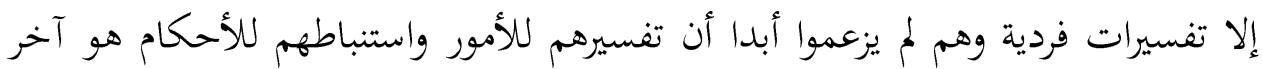

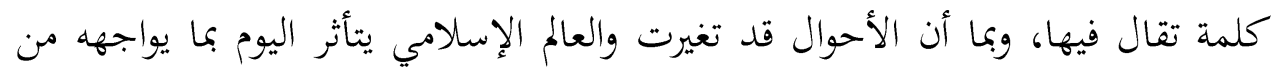

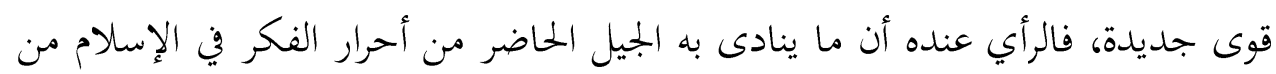

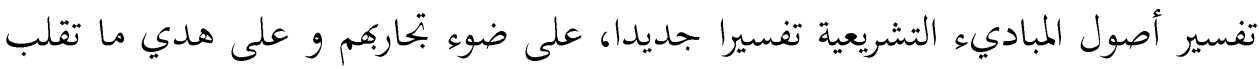
على حياة العصر من أحوال متغايرة هو رأي له ما يسوغه كل التسويغ. 24

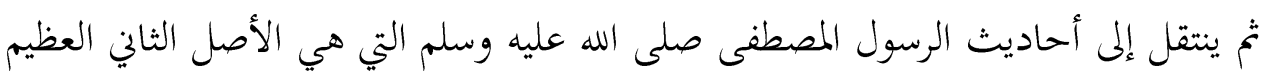

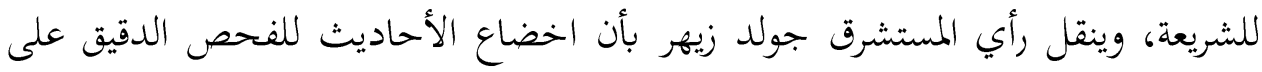

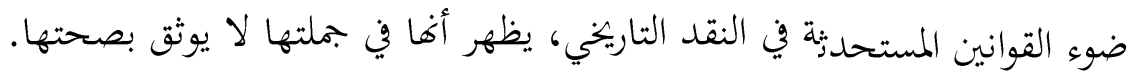

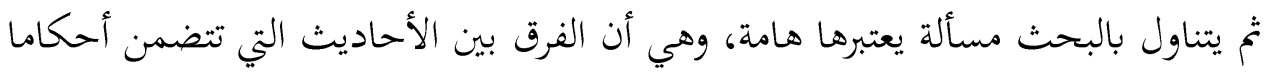

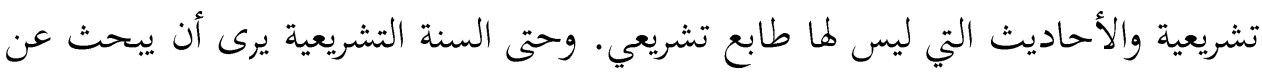

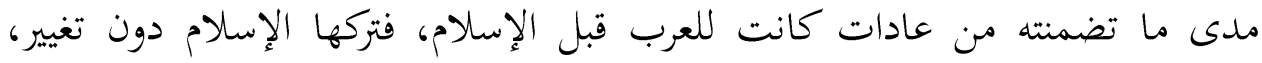

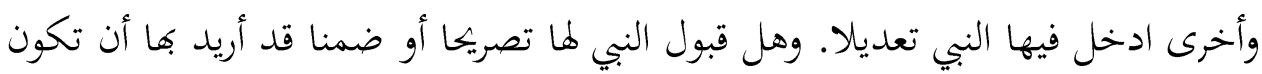
ذات صفة عامة في تطبيقها.

${ }^{24}$ Iqbal Sheikh, Iqbal Nama, Majmooa' Makaatib Iqbal, Lahore, P. 48-52; Bistami, Muhammad Saeed, Mafhhom Tajdid Din, P. 139-140. 
ويستشهد بأن أباحنيفة لم يكن أحيانا يعتمد على هذه الأحاديث، وذلك في نظره موقف

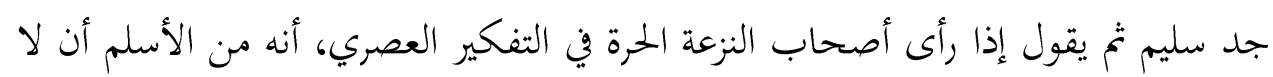
تتخذ هذه الأحاديث من أدنى تفريق بينها، أصلا من أصول التشريع بين أهل السنة.

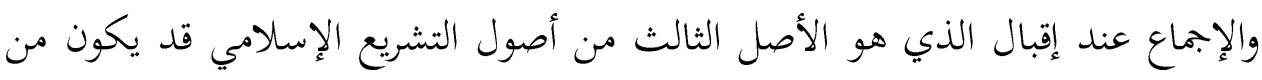

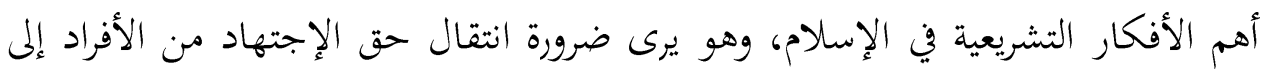

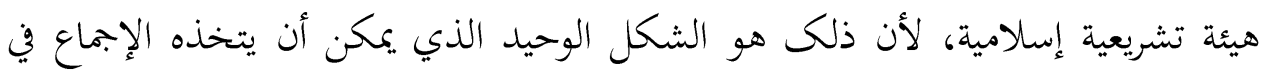

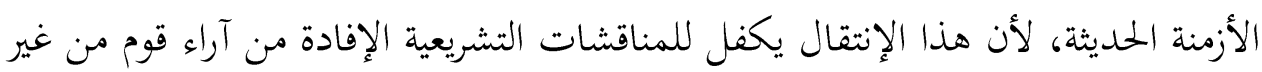

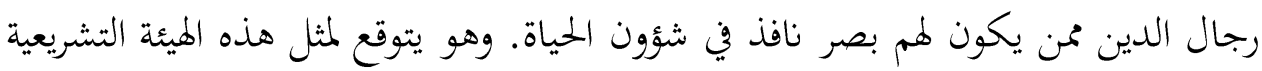

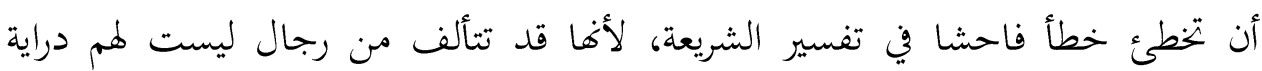

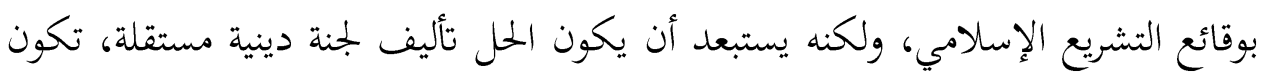

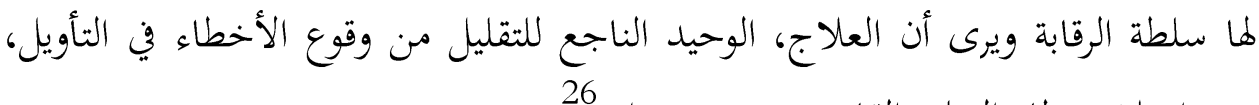
وهو إصلاح نظام التعليم القانوني وتوسيع مداه.

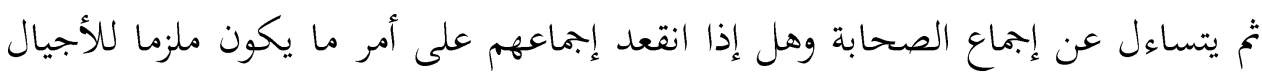

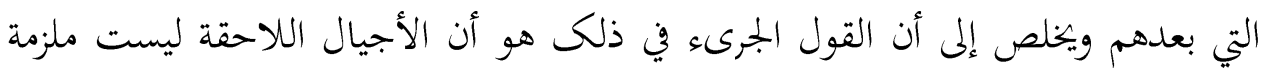
بإبماع الصحابة.

والأصل الرابع من أصول الفقه هو قياس ويرى إقبال أن القياس كان في الأصل ستارا

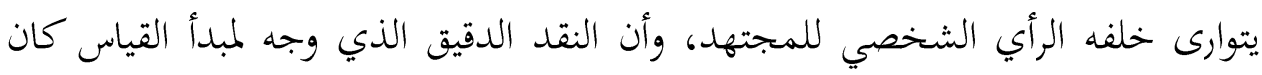

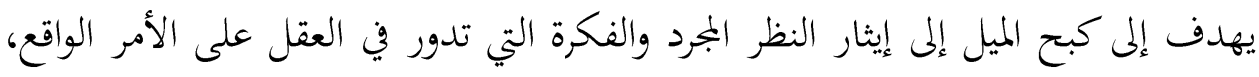

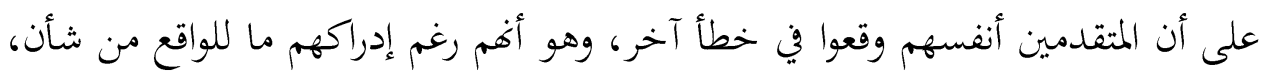

${ }^{25}$ Ibid. 48-52.

${ }^{26}$ S.M. Yousuf: A Study Of Iqbals Views On Ijma, Iqbal Review, 3.3-OCT 1962, P.17-25. 
إلا أفم في الوقت نفسه جعلوه ثابتا إلى الأبد وقصروا نظرهم على السابقات التي وقعت

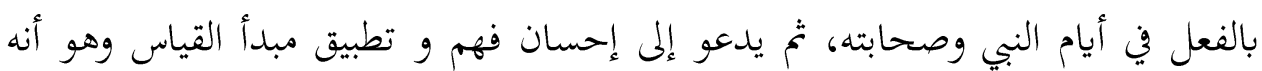
27

\section{حق طليق في حدود النصوص الملزمة. 4.1}

يقول عن الإجتهاد: ولا ريب عندي في أن التعمق في درس كتب الفقه والتشريع الهائلة العدد، لا بد أن يجعل الناقد بمنجاه من الرأي السطحي الذي يقول بأب أنس شريعة الإسلام

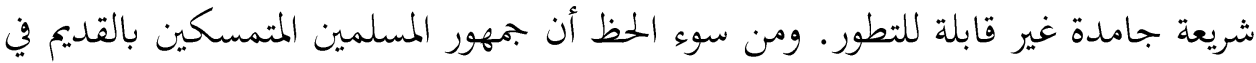

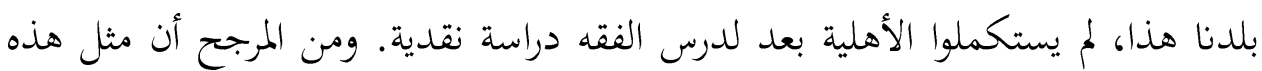

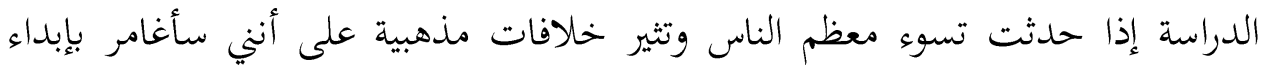
بعض الملاحظات عن النقطة التي نبحثها الآن:

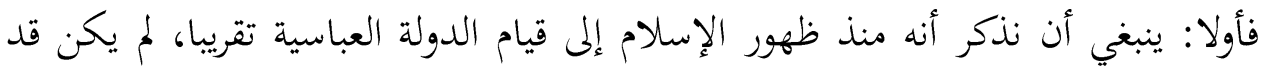
دون شرائع الإسلام سوى القرآن.

ثانيا: مما هو جدير بالملاحظة أنه منذ حوالي منتصف القرن الرابع الهجري، ظهر مال لا

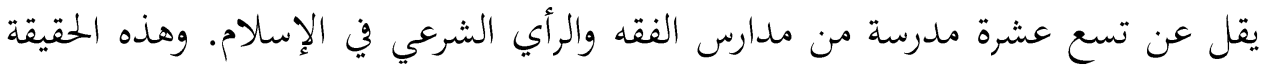

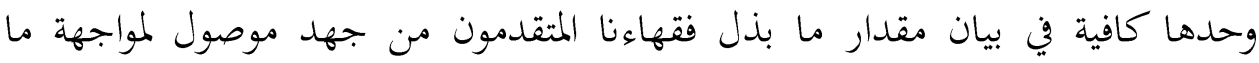

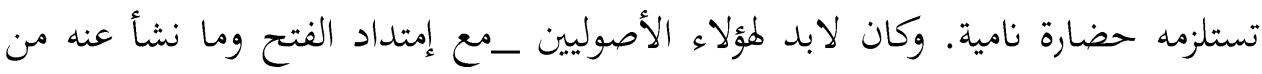

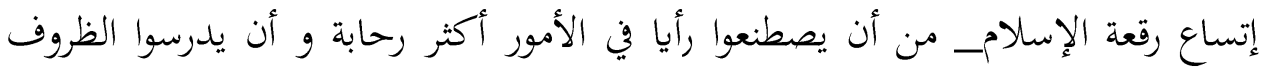
الخحلية للحياة وعادات الشعوب الجديدة التي دخلت في حظيرة الإسلام. والدرس الدقيق لمدارس الرأي الفقهي على إختلافها، في ضوء التاريخ السياسي والإجتماعي المعاصرين،

${ }^{27}$ Bistami, Muhammad Saeed, Mafhoom Tajdeeduddin, P. 141-149. 
يكشف عن أن المشرعين تحولوا شيئا فشيئا عن طريقة الإستنباط في تأويلهم إلى طريقة

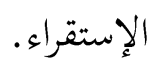

ثالثا: عندما ندرس أصول الفقه الإسلامي الأربعة المتفق عليها، وما ثار حولها من خلاف،

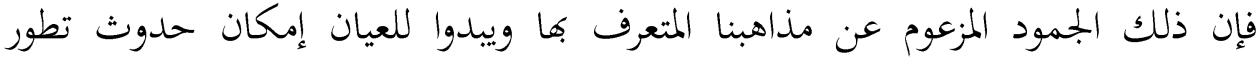

والآن إذا كانت عقيدة ختم الرسالة حافزة للإنسان المعتقد بها على عدم الإنتظار والترقب،

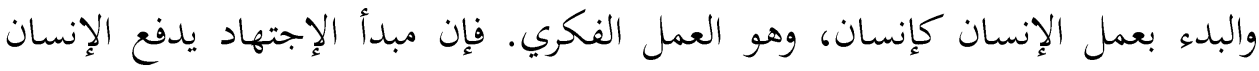

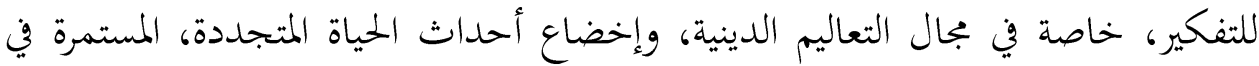
التجدد، وتكييفها بكيفية إسلامية. 29

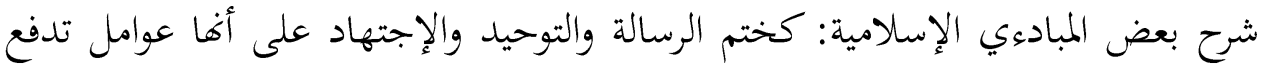

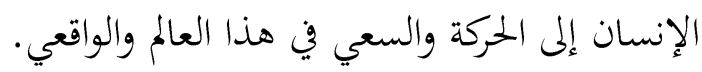
وإذن ييغي إقبال من إصلاح الفكر الديني في الإسلام، أن تعود القوة للمسلم وأن يرى قوته هذه ليست في إتباع فلسفة من فلسفات الغرب بل في فهم الإسلام فهما صحيحا،

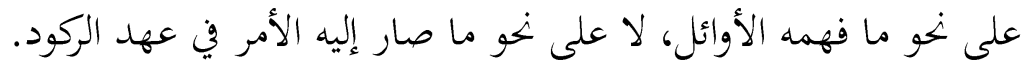

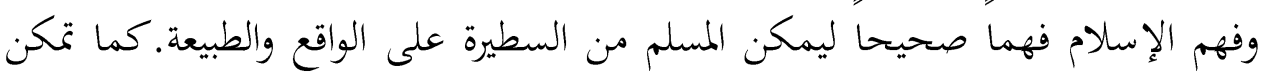

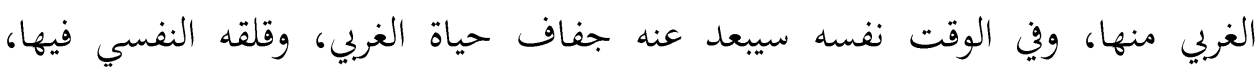
وسيجعله أكثر إلماماً بالحقيقة.

${ }^{28}$ Al-Bahi, Muhammad, Al-fikr Islami Al-hadith, p. 411-412.

${ }^{29}$ Ibid. p. 398-399.

${ }^{30}$ Ibid. p. 399-413. 
وعلى هذه الأسس يدعو إقبال العالم الإسلامي أن يقدم بشجاعة على إتمام التجديد الذي

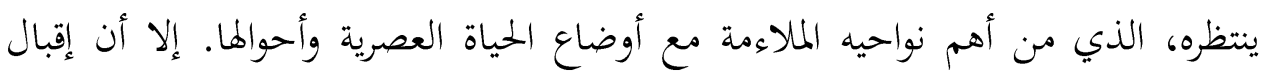

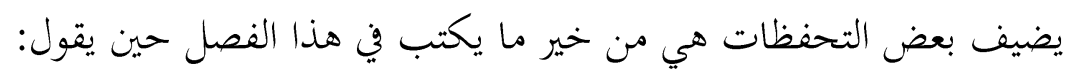

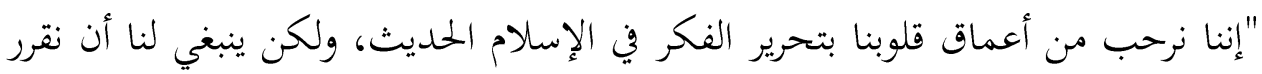

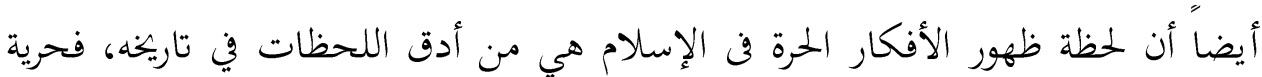

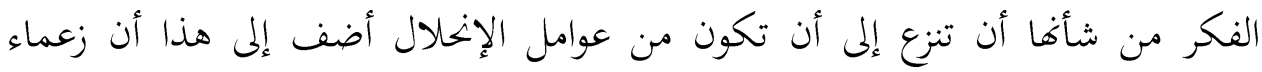

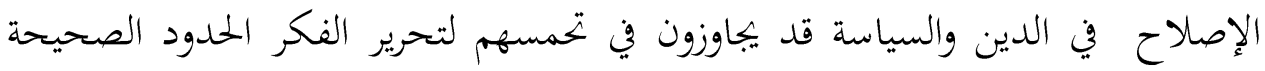

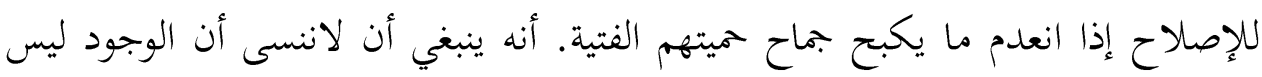

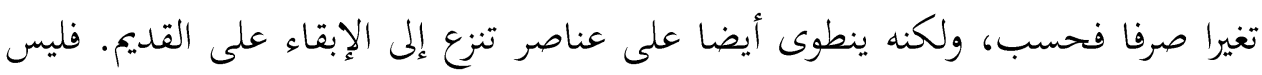

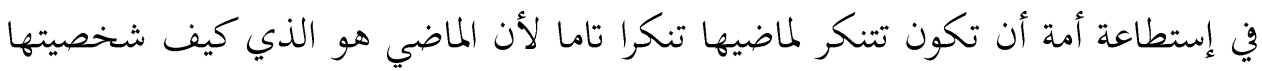
الحاضرة.

\section{5. اصلاصة الحث:}

يتضح من خلال العرض آراء ووجهات نظر رجال فكر والإصلاح العصرانيين قيد الدراسة

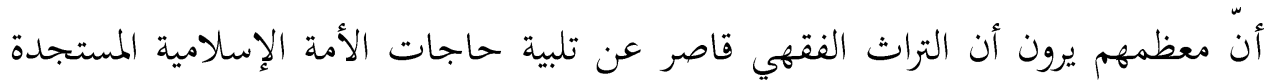

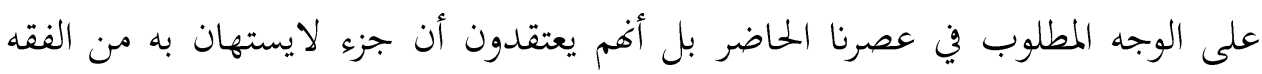

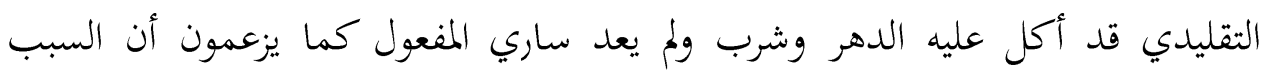

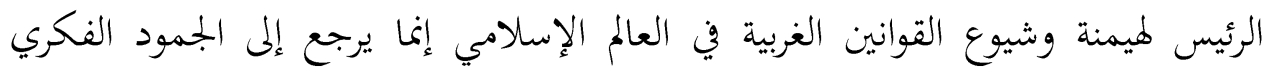
ولتجحر العقلي لدى العلماء المسلمين وإغفالهم جانب الإجتهاد.

${ }^{31}$ Bistami, Muhammad Saeed, Mafhhom Tajdid Din, P. 141-142. 
وطمعا في تطبيق الشريعة الإسلامية في مناحي الحياة المختلفة الإجتماعية منها وسياسية والإقتصادية للأمة الإسلامية فهم يؤكدون على ضرورة إعادة صياغة الفقه الإسلامي من

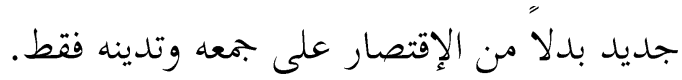
ولذا فهم يلحون على إعادة النظر في مصادر الفقه الإسلامي المعروفة، إضافة إلى تأييدهم لفكرة الإجتهاد الجماعي ويريدون بذلك تحرير مهمة الإجتهاد من إحتكار العلماء وأهل الفقه التقليديين ويقترحون إسنادها للمجالس التشريعية في البلاد. ولا يمكن إنكار ما لعبت آراء هؤلاء الزعماء من دور مشكور في توعية الشعوب وإيقاظ الهمم في المجالات العلمية والفكرية وتوجيه العلماء التقليديين في العالم الإسلامي نحو ضرورة إعادة صياغة القانون الإسلامي عن طريق إتباع منهج الإجتهاد الجماعي وكان لهذا النداء صراء في العالم الإسلامي حيث انشئت نتيجة لذلك بجامع فقهية تقوم بدورها في التوسل إلى إيجاد حلول للمشاكل التي يواجهها المسلم المعاصر.

\section{(C) (1)(@)}

This work is licensed under a Creative Commons Attribution-NonCommercial-ShareAlike 4.0 International (CC BY-NC-SA 4.0) 